The feet are lifted too high or not high enough, one leg is thrown out from its line of march or across the path of its fellow, the feet are replaced on the ground in the wrong place and often with a thud. Walking may become quite impossible without any weakness being present.

Inco-ordination of a different kind is seen in cerebellar lesions. The afferent pathways are intact but the orderly execution of simultaneous and successive movements is impaired. The legs are held wide apart to give a broader base, one or both legs tends to deviate to right or left of its line of march. Any slight tendency to lose balance is corrected late and with difficulty and often too violently, and the gait becomes staggering and "drunken" in quality.

Such inco-ordination of gait is met with in chronic disease of the cerebellum such as tumours or degenerations, in degenerations of the cerebellar tracts as in Friedreich's disease and the other hereditary ataxies. In acute cerebellar lesions the ataxy is usually too intense to permit even an attempt at walking.

In many nervous diseases the associated movements seen in normal progression are diminished. In hemiplegias and in Parkinsonian states the arm instead of swinging naturally is held stiff and still. In cerebellar disorders and in chorea the affected arm is seen to hang passively and to swing like a pendulum under the influence of gravity rather than by its own associated movement.

In other cases involuntary movements are seen. The rhythmic tremor of paralysis agitans, the irregular jerky movements of chorea, the head nodding of Friederich's disease and the squirming movements of athetosis seen in juvenile hemiplegia may all be accentuated during the act of walking.

The foregoing are some of the pieces of information that can be elicited from a patient with nervous disease at first sight and before any history has been taken or any detailed examination made. They may be of inestimable value, but the method of quick diagnosis should be used with great caution, and first impressions should be discarded without hesitation if they will not stand the test ot careful examination and reasoned criticism.

\title{
DEFORMITIES OF THE FOOT IN CHILDREN.
}

\section{The Treatment of those Types due to Affections of the Nervous System.}

\section{BY B. WHITCHURCH HOWELL, F.R.c.s.}

THE following types will be discussed in this article :-

(I) Anterior poliomyelitis.

(2) Spastic paralysis.

(3) Fredreich's ataxia.

(4) Pseudo-hypertrophic muscular paralysis.

(1) Anterior Poliomyelitis.

The clinical picture of the acute stage is too well known to be enlarged upon. The cardinal principles of treatment are to rest the weak or paralysed muscles in the position of optimum utility, preferably in simple splints in the prone position, or in plaster of 
Paris. Serum obtained from patients who have passed through the disease is of great value, as was proved by the writer in an extensive epidemic in England in 1926.

The orthopædic masseuse has many weary weeks or months of treatment by massage, electricity and muscle re-education, and such may have to be continued even for two years.

Should deformities of the foot then persist these may be due to one of three factors.

(I) Loss of muscle balance due to paralysis of one group of muscles, e.g., talipes equinovarus from paralysis of the peronei.

(2) Loss of muscle balance due to complete paralysis of the extrinsic muscles, e.g., flail foot, with or without an associated varus or valgus deformity.

(3) Bony deformities associated with either of the above groups.

Treatment of Deformities.-(I) In the first class a transplantation of tendon should be performed. If the peronei be paralysed then the tendon of the tibialis anticus is transferred, with a portion of its bony insertion, into a bed prepared for it in the base of the fifth metatarsal bone. The important technical points in this operation are : first a bony insertion, second the muscle and tendon must act along a long straight line without friction, and finally the orthopædic masseuse must re-educate the muscle from the fourth day after the operation. Within a few weeks strong voluntary eversion of the foot is thereby obtained.

It cannot be stressed too emphatically that this particular tendon-transplantation is the only one of any value in the foot; all others are useless and should never be performed.

(2) In flail foot arthrodesis is resorted to in order to stabilize the foot and to maintain permanently a good shape for the foot.

In certain instances a bony block may be built up posterior to the ankle-joint to act as a stop to excessive foot drop. This operation has its merits in picked cases, having passed out of the experimental stage.

The operative formation of a neat foot saves much expense in the provision of boots and appliances, and often ready-made boots and shoes can then be worn with or without simple alterations.

On the other hand, if talipes calcaneo-valgus be under consideration, there are two courses open to the orthopædic surgeon-one, the operation invented by Whitman, of removal of the astragalus, and backward displacement of the foot; and the other, arthrodesis of the subastragaloid joints with a bone-block anterior to the ankle-joint. In certain cases an arthrodesis of the ankle-joint itself is performed, especially when the intrinsic muscles of the foot are strong.

(3) In certain bony deformities the operations described above are carried out in series; the tendon transplantation being done at a later date, to get the best results.

In no instance should operations on bone be performed in patients under ro years of age, and in all cases great discrimination is needed, especially in the treatment of talipes calcaneus, and the more experienced the orthopædic surgeon, the fewer and simpler become the operations which he performs on the paralytic foot.

\section{(2) Spastic Paralysis.}

The diagnosis of such cases is usually simple. Soon after birth the child's limbs are noticed to be stiff, straight and spastic, the lower limbs being in the position of 
adduction with contraction of the adductors, the knees straight or slightly bent, and the feet in the position of talipes equinus, talipes equinovarus, or talipes calcaneo-valgus. The upper limbs also are affected, either one or other, the deltoid being in spasm, the elbow contracted, the hand and fingers flexed. There are certain sub-divisions of this type of spastic paralysis, namely, spastic hemiplegia affecting one side; or spastic diplegia of the lower limbs; or spastic tetraplegia, affecting all four limbs. Since deformities of the lower limb are to be discussed, these may be dealt with along the following lines :-

(I) Tenotomy of the affected contracted tendons.

(2) Stoffel's operation on the nerves supplying the groups of spastic muscles.

(3) Re-education in walking.

(4) Special boots, with or without heels, according to the severity of the case.

The treatment in simple cases is by subcutaneous tenotomy of the adductors and the tendo Achillis.

The foot is corrected and placed in the plantigrade position in plaster of Paris for a minimum period of four to six weeks, after which a metatarsal bar with or without a low heel is worn in the boot in order to teach the child to dorsiflex the foot. It is very seldom necessary to perform tenotomies upon the ham-strings for contraction of the knee; if so, it need hardly be stressed that such an operation must be an open one, since there is a grave risk of dividing the external popliteal nerve as it lies immediately under the tendon of the biceps.

In a few instances a walking calliper or a simple leg iron with a $T$-strap may be necessary. This should be worn for a short time after the operation to enable the patient to learn to walk without contraction of the lower limb.

Stoffel's operation on the peripheral nerves should be reserved for more advanced cases of deformity, the nerve which supplies the contracted ham-strings, the contracted gastrocnemius, \&c., the sciatic nerve and its branches being explored. A percentage of the nerve-fibres going to each spastic group are divided in such a way that regeneration cannot possibly take place. Experience and knowledge of the degree of the spasticity will enable the surgeon to judge what percentage should be thus divided.

Needless to say an organic contracture of the tendon must be dealt with by elongation, with, if necessary, Stoffel's operation on the nerve supply.

This particular operation in selected cases, specially in the upper limb, is one of the most dramatic of nerve surgery, the results, with re-education being extraordinarily good. It cannot however be stressed sufficiently that it will not remedy a contracture of a tendon which is left untreated surgically by elongation.

The after-treatment of the deformity after this operation is identical with that of subcutaneous tenotomy, the patient being under the care of an orthopædic masseuse who trains the child to dorsiflex and evert the foot when starting to walk. Later on the child walks with a metatarsal bar and a low heel so that every step taken reminds the child to dorsiflex the foot. Should, however, the case be seen later on in life when further deformities have developed, such as contractures of the soft parts, or even deformities of bone and joint, then more radical surgical measures will be needed.

The plantar fascia may have to be divided either with a tenotome or preferably by Steindler's operation, which has been modified by the writer, so that an adequate 
exposure of the intrinsic muscles of the foot at their origin from the tubercle of the os calcis can be obtained.

The prognosis of cases dealt with surgically are good, provided: there are no mental defects, there is good control of the excreta, fits, if existent, can be dealt with and controlled by drugs, and lastly, that athetosis is not marked.

\title{
(3) Friedreich's Ataxia.
}

In the majority of cases in which ataxia and fits are associated patients can be taught to walk and become less ataxic with fewer fits if the foregoing operations are carried out.

The teaching of the older surgeons was that patients with fits and athetosis were totally unsuitable for surgical intervention. The modern view is that provided the fits can be controlled surgical intervention is of great value, the child's mind developing as the result of being able to walk.

\section{(4). Pseudo-hypertrophic Muscular Paralysis.}

The prognosis in such cases is exceedingly unfavourable. The classical picture of such a paralysis is well known. The calves are fixed and abnormally large and hard. The child finds it difficult to walk, the knees are contracted, and there is an equinus deformity of the foot with a scuttling gait, and for this reason the orthopædic surgeon's help is often required. A simple subcutaneous tenotomy of the tendo Achillis will often enable the patient to walk-particularly if after the tenotomy the lower limbs are placed in Thomas' splints for some four to six weeks, during which time the knees are carefully and firmly bandaged into position of dead extension.

Should deformities be more marked more radical operations are necessary similar to those already described.

Surgical treatment is of great value. It cannot be emphasized too st:ongly that simple operations can often enable the patient to continue to walk and get about for a long time, before he becomes bedridden and succumbs to the disease at the age of early adolescence.

\section{A CASE OF LOW BLOOD-PRESSURE TREATED WITH PITUITARY WHOLE GLAND BY THE MOUTH.}

\author{
BY BERNARD MYERS, C.M.G., M.D.
}

ARTERIaL blood-pressure is dependent upon four factors: the contracting force of the left ventricle, the volume of blood propelled by the contraction into the full arteries, the elasticity of which the middle coats of the large arteries are capable, and the peripheral resistance.

The systolic blood-pressure is synchronous with the systole of the ventricle and the diastolic with the diastole. By subtracting the diastolic from the systolic blood-pressure we obtain the figure representing the pulse-pressure. The figures obtained are far from being absolutely fixed ones, as the same individual may show quite definite changes during the twenty-four hours or even in a few minutes if subjected to stress or excitement, or again, on the other hand, during sleep. It is a good plan to take the blood-pressure at least an hour after exercise and before a meal. 